Verbrennung von Treibmitteln mit $\mathrm{NO}_{2}$-gebundenem Sauerstoff unter höheren Drücken (Raketen, Geschütz) mit diesen Reaktionen zu rechnen ist, auch wenn die Banden der zugehörigen $Z$ wischenkörper nicht zu verzeichnen sind. Die Erhöhung des Druckes bringt jedoch vor allem eine Begünstigung des Ablaufes von Dreierreaktionen, die die Verbrennung mit steigendem Druck vollständiger in Richtung der Bildung von elementarem Stickstoff gestalten, wodurch lediglich der Umsatz über die Reaktionen (I) und (II) anteilmäßig, aber nicht in ihrer absoluten Höhe, beeinträchtigt wird. Untersuchungen der Verbrennung ron
Treibmitteln bei Normaldruck sind deshalb besonders geeignet, den Ablauf von Zweierstoß-Reaktionen hervortreten zu lassen, die bei höherem Druck relativ in den Hintergrund treten, jedoch auch dort für manche Verbrennungseigenschaften von wesentlicher Bedeutung sind. Nicht näher berücksichtigt wurden in dieser Arbeit wegen der experimentellen Schwierigkeiten in der Handhabung Brennstoffgemische mit $\mathrm{HNO}_{3}$ als Sauerstofflieferant; da jedoch auch hier der Sauerstoff $\mathrm{NO}_{2}$-gebunden vorhanden ist, dürften unsere Ergebnisse auch in den Hauptzügen für diese Gemische gelten.

\title{
Über neue Spektren in der Glimmentladung mit Benzoldampf
}

\author{
Von H. SchüLer und L. Reinebeck
}

Aus der Forschungsstelle für Spektroskopie in der Max-Planck-Gesellschaft, Hechingen (Z. Naturforschg. 6 a , 16C-165 [1951]; eingegangen am 8. Januar 1951)

\begin{abstract}
Während die Änderung der Anregungsbedingungen bei Atomen das Verhältnis von Bogenzu Funkenspektren variiert, treten bei vielatomigen organischen Molekülen außer der arteigenen Molekülemission auch Spektren auf, die Bruchstücken des Moleküls zugeordnet werden müssen. Die Methode, wie man in der positiven Säule einer Glimmentladung die Anregungsbedingungen ändern kann, ist in einer kürzlich erschienenen Arbeit ${ }^{1}$ beschrieben worden.

Bei einer derartigen Untersuchung an Benzol erscheinen eine Reihe von Spektren, von denen vier bisher unbekannt waren. Diese Spektren sind isolierbar, weil jedes von ihnen nur unter bestimmten Anregungsbedingungen erscheint. Das Auftreten dieser Spektren ist vermutlich so zu verstehen, daß das Benzol je nach den Anregungsbedingungen in verschiedene Bruchstücke zerfällt, die durch ihr Leuchten nachgewiesen werden. Die Träger der vier neuen Spektren selbst lassen sich noch nicht definitiv angeben.
\end{abstract}

$\mathrm{I}_{\mathrm{v}}^{\mathrm{n}}$ n einer kürzlich veröffentlichten Arbeit ${ }^{1}$ haben die Verfasser gezeigt, daß sich in der positiven Säule einer Glimmentladung die Anregungsbedingungen einer organischen Substanz in gewissen Grenzen variieren lassen. $\mathrm{Da}$ die nachstehende Arbeit eine Fortsetzung der dort mitgeteilten Überlegungen ist, seien diese als bekannt vorausgesetzt. Die Methode ist etwa folgende: Brennt man eine Glimmentladungsröhre mit reinem Helium, so stellt sich in der positiven Säule eine Elektronengeschwindigkeitsverteilung ein, die durch 19,81 eV (1. Anregungszustand) und $24,58 \mathrm{eV}$ (Ionisierungsgrenze) charakterisiert ist. Diese Geschwindigkeitsverteilung wird nun geändert, wenn man Moleküle hineinverdampft, die andere charakteristische $\mathrm{eV}$-Werte besitzen. Bei hohem Dampfdruck der Moleküle wird sich schließlich die Elektronengeschwindigkeitsverteilung einstellen, die deren eigenen eV-Werten entspricht, d. h. also, bei konstantem Heliumdruck wird die Elektronengeschwin- digkeitsverteilung von der Menge der zugesetzten Moleküle abhängig sein. Damit werden die Anregungsbedingungen für die Untersuchungssubstanz durch deren Dampfdruck variierbar.

Bei Versuchen dieser Art stellt sich nun heraus, daß nicht etwa nur ein Molekülspektrum erscheint, das sich mit den Anregungsbedingungen ändert, sondern es treten verschiedene Bandenspektren auf, deren Erscheinen an bestimmte Dampfdruckbereiche gebunden ist.

Im folgenden sollen für den Fall von Helium und Benzol die Befunde beschrieben werden. Da bei Benzol die charakteristischen eV-Werte $(4,72 \mathrm{eV}$ $=1$. angeregter Singulett-Zustand ${ }^{*} ; 9,19 \mathrm{eV}=$ Ionisierungsgrenze) wesentlich tiefer als bei der reinen Heliumentladung liegen, wird sich bei schrittweiser

1 H. S c h ü l e r u. L. R e i n e b e c k, Z. Naturforschg. 5 a, 657 [1950].

* Der tiefste Triplett-Zustand liegt bei $3,63 \mathrm{eV}$. 


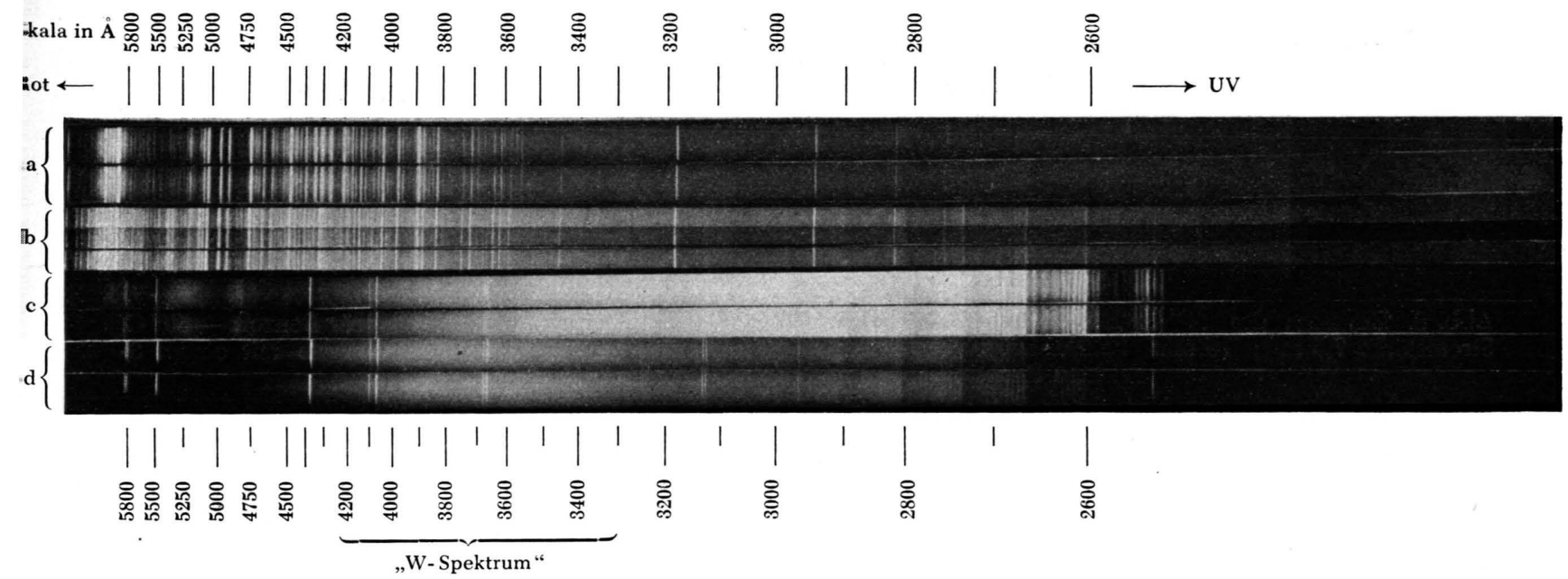

Abb. 1. Emissionsspektren einer Glimmentladung von Helium mit Benzoldampf. He-Druck konstant einige Zehntel mm, Benzoldampfdruck variabel.

Temperatur am Verda
$\mathrm{a}-70^{\circ} \mathrm{C}$
$\mathrm{b}-50^{\circ} \mathrm{C}$
$\mathrm{c}-33^{\circ} \mathrm{C}$
$\mathrm{d}+20^{\circ} \mathrm{C}$

Stromstärke

$10 \mathrm{~mA}$

$7,5 \mathrm{~mA}$,

$20 \mathrm{~mA}$

$60 \mathrm{~mA}$

Kontaktkopien von Aufnahmen mit mittlerem Hilger-Quarz-Spektrographen. Die Spektren b, c, d enthalten Hg-Vergleichslinien. Platte: Perutz peromnia. Bei der Reproduktion sind von jeder Druckstufe je 2-3 Spektren aneinandergefügt, da die Originalspektren sehr schmal sind.

Erhöhung des Benzoldampfdruckes die Elektronengeschwindigkeitsverteilung allmählich nach niedrigeren Werten zu verschieben.

In Abb. 1 ist eine solche Versuchsreihe wiedergegeben. Als Untersuchungssubstanz wurde Benzol, p. a., von der Firma Merck, Darmstadt, benutzt. Die Spektren sind mit einem mittleren HilgerQuarzspektrographen aufgenommen. Es sind immer solche Druckstufen gewählt worden, bei denen eine merkliche Änderung des Leuchtens festgestellt werden konnte.

1. Wenn man zunächst Benzol nur spurenweise einer Heliumentladung zusetzt, dann werden diese Moleküle mit wesentlich höheren Elektronengeschwindigkeiten angeregt, als es in der reinen Benzolentladung der Fall ist. In Abb. 1, Spektrum a, ist dieses Stadium aufgenommen bei einer Benzoltemperatur von $-70^{\circ} \mathrm{C}$ am Verdampfungsort, die einem Benzolsättigungsdampfdruck von einigen Hundertstel $\mathrm{mm}$ entspricht ${ }^{2}$. Bei den vorliegenden Versuchen beträgt der Druck des Heliums im allgemeinen einige

2 Wie in der früheren Arbeit erwähnt, stellen diese Dampfdruckangaben einen Maximalwert dar, weil der Benzoldampf ja durch die positive Säule nach den Kühlfallen (in flüssigem Stickstoff) hinströmt, wo er sich in festem Zustand niederschlägt.
Zehntel mm. Unter diesen Anregungsbedingungen sieht man neben einem sehr starken Heliumspektrum die Spektren von $\mathrm{C}_{2}, \mathrm{H}_{2}$ und $\mathrm{CH}$. Die Anwesenheit von $\mathrm{C}_{2}$ wird durch die Swan-Banden (0,0-Bande $5165 \AA)$ nachgewiesen. $\mathrm{H}_{2}$ macht sich vor allem durch seine starken Banden im Roten bei $5800-6100 \AA$ bemerkbar; das kontinuierliche $\mathrm{H}_{2}$-Spektrum ist ebenfalls, allerdings relativ schwach, zu erkennen. Das Auftreten der Bande $4313 \AA$ deutet darauf hin, daß auch $\mathrm{CH}$ in der Entladung entsteht. Da die normale Emission des Benzolringes in der Gegend von 2600 bis $3000 \AA$ nicht zu beobachten ist, muß man schließen, daß die Benzolmoleküle vollständig zerstört werden, wenn sie mit den Elektronengeschwindigkeiten der Heliumentladung oder durch den metastabilen Heliumzustand bei 19,81 eV angeregt werden.

Während man bei Energieübertragung von Helium auf geringe Beimischungen von Fremdatomen oder von 2-atomigen Molekülen (z. B. CO) das Leuchten ionisierter Zustände beobachtet (Funken-Spektren), führt sie bei organischen vielatomigen Molekülen, wie hier beim Benzol, zu einer vollständigen Zerstörung der Moleküle.

2. In Abb. 1, Spektrum b, ist nun der Dampfdruck des Benzols etwas erhöht worden $(\sim 0,3 \mathrm{~mm}$ am Ver- 


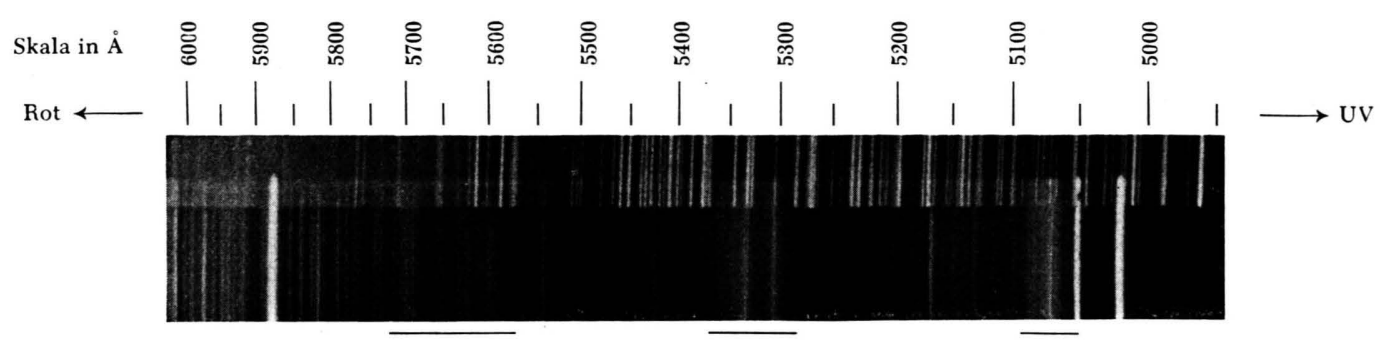

Abb. 2. Emission des „T“-Spektrums. He-Druck einige Zehntel mm, Temperatur des Bades für Benzol: $-50^{\circ} \mathrm{C}$. Stromstärke 7,5 mA; $15 \mathrm{~min}$ belichtet. Fe-Vergleichsspektrum; Platte: Perutz peromnia. Aufnahme mit einem 3Prismen-Spektrographen nach Zeiß-Försterling; $f=27 \mathrm{~cm}$.

dampfungsort). Auch hier sind die Heliumlinien noch stark sichtbar, haben aber in ihrer Gesamterscheinung gegen Abb. 1, Spektr. a, etwas abgenommen. Neben $\mathrm{C}_{2}, \mathrm{H}_{2}$ und $\mathrm{CH}(\mathrm{CH}$ ist etwas intensiver geworden als in Abb. 1, Spektr. a) erscheinen 2 oder 3 neue Bandengruppen im Sichtbaren. Diese Bandengruppen sind in Abb. 2 (nach Aufnahmen mit größerer Dispersion mit einem 3-Prismenspektrographen nach ZeißFörsterling) wiedergegeben. Sie liegen bei 5060 bis $5080 \AA, 5300-5350 \AA$ und bei $5600 \AA$, wobei die $\mathrm{Zu}-$ gehörigkeit der dritten Gruppe bei $5600 \AA$ noch nicht gesichert ist, da sie als die schwächste Gruppe durch die überlagerten Wasserstofflinien besonders wenig hervortritt.

Diese Bandengruppen werden deshalb als zusammengehörig betrachtet, weil sie immer gleichzeitig auftreten und sich ihr Intensitätsverhältnis zueinander nicht ändert. Soweit den Verfassern bekannt, sind diese Banden bisher nie erwähnt worden. Sie treten am klarsten bei kleinen Stromstärken (3-7,5 mA) hervor, während sie bei größeren Stromstärken (10 mA und höher) schon merklich an Intensität verlieren. Ihr Erscheinen ist an ein ziemlich eng begrenztes Mischungsverhältnis von Helium + Benzol gebunden. Es gehört also eine ganz bestimmte Elektronengeschwindigkeitsverteilung dazu, um im Benzol diesen Träger herzustellen und anzuregen.

Über den Träger selbst läßt sich zunächst wenig aussagen. Es sei nur bemerkt, daß beim Naphthalin, Diphenyl und Diphenylmethan $\left(\mathrm{C}_{6} \mathrm{H}_{5}-\mathrm{CH}_{2}-\mathrm{C}_{6} \mathrm{H}_{5}\right)$ unter ähnlichen Mischungsverhältnissen mit Helium auch diese Banden beobachtet werden. (Versuche mit weiteren Substanzen liegen bisher nicht vor.) Vermutlich handelt es sich bei dem Träger um ein Molekülbruchstück mit mehr als 2 Atomen, da bei den hier vorhandenen Atomen, $(\mathrm{C}, \mathrm{H}, \mathrm{He})$ das Leuchten der 2-atomigen Verbindungen unter den vorliegenden Versuchsbedingungen bekannt ist und nicht mit dem neuen Spektrum übereinstimmt. Zur Unterschei- dung von den weiter unten diskutierten neuen Spektren sei dieses als „T-Spektrum“ bezeichnet. Außerdem ist in Abb. 1, Spektr. b, im Ultraviolett (Gegend 2600 — $3000 \AA)$ die bekannte Emission des unzerstörten Benzolringes schwach angedeutet.*

Die Ergebnisse der Abb. 1, Spektr. a und b, lassen sich zusammenfassend etwa so beschreiben: In Abb. 1, Spektr. a, tritt eine vollständige Zerstörung des Benzolmoleküls ein. Durch die Dampfdruckerhöhung in Abb. 1, Spektr. b, findet eine Verschiebung der Elektronengeschwindigkeiten nach kleineren Werten hin statt, die sich in dem Auftreten größerer Molekülbruchstücke ausdrückt; auch sind die ersten Anzeichen von dem Leuchten unzerstörter Moleküle vorhanden.

Einen Anhaltspunkt für die Energie, die für das Auftreten des „T-Spektrums“ notwendig ist, geben Versuche, bei denen Benzol in eine Entladung von Quecksilberdampf gebracht wurde ${ }^{3}$. Die für die $\mathrm{Hg}$ Entladung charakteristischen eV-Werte sind $4,9 \mathrm{eV}$ (1. Anregung) und 10,4 eV (Ionisation), sie liegen nur wenig höher als die entsprechenden Werte einer reinen Benzolentladung (4,72 und 9,19 eV). Unter diesen Versuchsbedingungen erscheint das „T-Spektrum" nicht. Man wird also das Benzol in Entladungen von Gasen, deren eV-Werte höher liegen (z. B. $\mathrm{H}_{2}$ oder A), bringen müssen, um die Energiewerte für das Auftreten des „T-Spektrums“ näher festlegen zu können.

Nach diesen Anschauungen dürfte bei kleinen Elektronengeschwindigkeiten, wie sie bei Entladungen in Natriumdampf anzutreffen sind $(2,12 \mathrm{eV}$ $=1$. Anregung, $5,14 \mathrm{eV}=$ Ionisation), die Anregungsenergie für die hineinverdampften Benzolmoleküle

* Anm. b. d. Korr.: Aufnahmen mit einem Entladungsrohr, das eine kürzere positive Säule besitzt, zeigen das „T-Spektrum“ auch ohne die Emission des Benzols im Ultraviolett.

3 Näheres über die Verwendung von verschiedenen „Leitgasen“ zur Änderung der Anregungsbedingungen der Untersuchungssubstanz in der erwähnten Arbeit 1. 


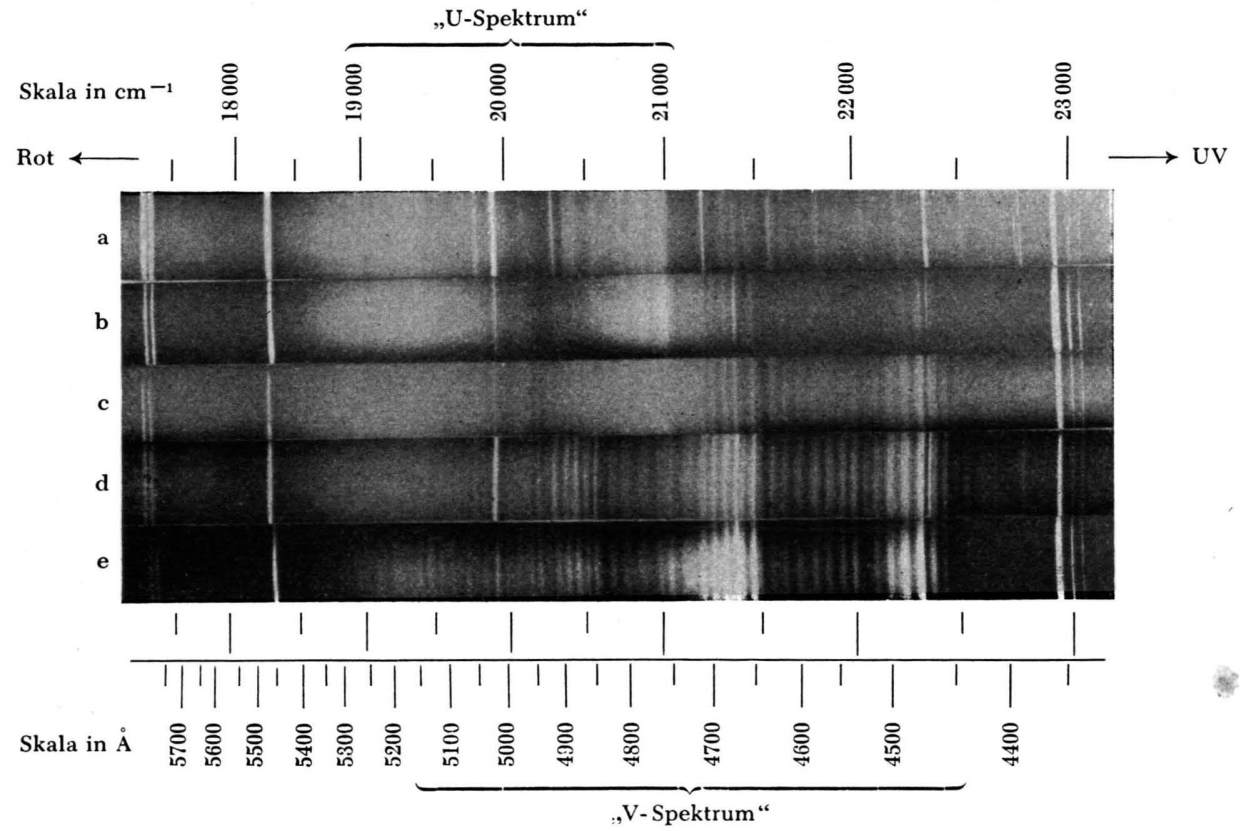

Abb. 3. Emission des „U“- und „V“-Spektrums. Aufnahme mit 3-Prismen-Spektrographen nach Zeiß-Försterling; $f==12 \mathrm{~cm}$. Platte: Perutz peromnia; Hg-Vergleichslinien. Bei a und d keine genaue Temperaturangabe, da nicht mit Bad, sondern mit Ofen geheizt und Temperatur am Verdampfungsort nicht genau festlegbar.

$$
\text { Substanz }
$$

\section{a}

Diphenyl $\mathrm{C}_{6} \mathrm{H}_{5} \cdot \mathrm{C}_{6} \mathrm{H}_{5}$

Benzol $\mathrm{C}_{6} \mathrm{H}_{6} \cdot \mathrm{C}_{6} \mathrm{H}_{5} \cdot{ }^{\circ} \cdot \cdot \cdot \cdot \cdot$

Benzol $\mathrm{C}_{6} \mathrm{H}_{3} \quad$. $\quad . \quad . \quad . \quad . \quad . \quad . \quad$.

Diphenylmethan $\mathrm{C}_{5} \mathrm{H}_{5} \cdot \mathrm{CH}_{2} \cdot \mathrm{C}_{6} \mathrm{H}_{5}$.

Toluol $\mathrm{C}_{6} \mathrm{H}_{5} \cdot \mathrm{CH}_{3}$. . . . . . . .

\section{Stromstärke

$$
\text { [mA] }
$$

12,5

10

20

12

10
Belichtungszeit

$20 \mathrm{~min}$

$15 \mathrm{~min}$

$21 \mathrm{~min}$

$60 \mathrm{~min}$

$30 \mathrm{sec}$
Temperatur am Verdampfungsort in ${ }^{\circ} \mathrm{C}$

$\sim+75$

$-35$

$-35$

$\sim+80$

$-53$ nicht ausreichen, um den Träger des „T-Spektrums“ zu erzeugen und anzuregen. Ein geringer Zusatz von Benzoldampf zu Na-Dampf sollte also kein „T-Spektrum" aufweisen; der Versuch bestätigt diese Erwartung.

3. In Abb. 1, Spektr. c, ist der Dampfdruck des Benzols weiter erhöht worden auf $\sim 1,4 \mathrm{~mm}$ Sättigungsdampfdruck am Verdampfungsort. Hier ist kein Leuchten des $\mathrm{He}$ mehr festzustellen. Die Elektronengeschwindigkeiten haben sich offensichtlich so weit erniedrigt, daß keine Anregung des $\mathrm{He}$ mehr erfolgt, obwohl in diesem Gasgemisch im Entladungsraum noch etwa gleich viele Heliumatome wie Benzolmoleküle vorhanden sind. Das Spektrum größter Intensität ist hier die UV-Emission des unzerstörten Benzolringes bei $2600-3000 \AA$. .

Im Sichtbaren hat sich das Leuchten vollkommen geändert. $\mathrm{C}_{2}$ und $\mathrm{CH}$ sind verschwunden; $\mathrm{H}_{2}$ ist nur in Spuren noch erkennbar; auch das "T-Spektrum “ ist nicht mehr zu beobachten. Dagegen tritt ein grünes Leuchten auf, das in seiner Gesamterscheinung nicht so intensiv ist wie das Leuchten im Sichtbaren bei Abb. 1, Spektr. a und b. In Abb. 3, Spektr. b und c, ist das sichtbare Spektrum gesondert wiedergegeben (nach Aufnahmen mit einem 3-Prismenspektrographen nach Zeiß-Försterling). Um die Erscheinung vollständig darzustellen, ist außer dem Spektrum b (der Abb. 3) eine stark überbelichtete Aufnahme (Spektr. c) eingefügt, um die Bandengruppen zwischen 4450 und $4750 \AA$ besser erkennen zu lassen. Das Leuchten setzt sich aus einer Reihe von scharfen Banden zwischen 4450 und $4750 \AA$ und 2 diffuseren Gruppen bei 4750 bis $4900 \AA$ und $5000-5400 \AA$ zusammen. Es läßt sich in seiner Gesamterscheinung durch Änderung der Stromstärke nicht beeinflussen.

Zunächst sollte man also annehmen, daß es sich um ein einheitliches Spektrum handelt. Versuche mit Benzolderivaten haben nun ergeben, daß es in Wirklichkeit zwei verschiedene Spektren sind. Die in Abb. 3, Spektr. a, wiedergegebene Aufnahme von Diphenyl $\left(\mathrm{C}_{6} \mathrm{H}_{5}-\mathrm{C}_{6} \mathrm{H}_{5}\right)$ zeigt ein Spektrum, das sich als identisch mit den diffusen Bandengruppen des Ben- 
zols erweist. Dieses so isolierte Spektrum sei im folgenden als „U-Spektrum“ bezeichnet. Die beiden Haupt-Maxima sind etwa $1500 \mathrm{~cm}^{-1}$ voneinander entfernt. Eine Bildung von Diphenyl in der Benzolentladung ist unwahrscheinlich, weil man sonst die wesentlich stärkere Diphenylemission im UV gleichzeitig beobachten müßte; es ist aber kein Anzeichen davon vorhanden.

Versuche mit Toluol $\left(\mathrm{C}_{6} \mathrm{H}_{5}-\mathrm{CH}_{3}\right)$ und Diphenylmethan $\left(\mathrm{C}_{6} \mathrm{H}_{5}-\mathrm{CH}_{2}-\mathrm{C}_{6} \mathrm{H}_{5}\right)$ zeigen nun, daß man auch die scharfen Bandengruppen des Benzols im Gebiet 4450—4i50 ̊̊ getrennt erhalten kann (Abb. 3, Spektr. $d$ und e). Dieses Spektrum sei mit dem Buchstaben „V“ bezeichnet. Man sieht auf Abb. 3, Spektr.d und e, daß das „V-Spektrum“ sich von $4750 \AA$ noch weiter nach Rot erstreckt, eine dritte Bandengruppe bei 4850-4950 $\AA$; dieser Teil ist beim Benzol aber von dem „U-Spektrum“ verdeckt. Das „V-Spektrum" tritt auch bei $\mathrm{C}_{6} \mathrm{H}_{5}-\mathrm{C}_{2} \mathrm{H}_{5} ; \mathrm{C}_{6} \mathrm{H}_{5}-\mathrm{C}_{3} \mathrm{H}_{7}(n)$; $\mathrm{C}_{6} \mathrm{H}_{5}-\mathrm{CH}_{2}-\mathrm{NH}_{2}$ und $\mathrm{C}_{6} \mathrm{H}_{5}-\mathrm{CH}_{2}-\mathrm{COOH}$ auf und ist in den früheren Arbeiten ${ }^{4}$ als sogenanntes „grünes“ Spektrum diskutiert worden. Aus dem „VSpektrum" kann man eine Hauptschwingung von $\Delta v \sim 940 \mathrm{~cm}^{-1}$ entnehmen; in dem Aussehen seiner Banden hat es Ähnlichkeit mit der Ringanregung des Benzols im Ultraviolett. Die Träger vom „U- und VSpektrum " können noch nicht endgültig festgelegt werden. Es ist nicht ausgeschlossen, daß in diesem Stadium das Leuchten von aromatischen Radikalen auftritt.

4. Erhöht man nun den Dampfdruck des Benzols in der Entladungsröhre noch weiter, dann verschwindet das grüne Leuchten der Spektren „U“ und „V“, ebenso wie beim Übergang von Abb. 1, Spektr. b, zu Abb. 1, Spektr.c, das „T“-Spektrum verschwand, dafür nimmt die positive Säule eine blaue Färbung an. Bei größeren Stromstärken (über $40 \mathrm{~mA}$ ) zeigt sich ein Kontinuum, das sich etwa von $3400-4400 \AA$ erstreckt und ein Maximum in der Gegend von $3700 \AA$ hat ${ }^{5}$.

In Abb. 1, Spektr. d, ist dieses Stadium wiedergegeben. Wie man sieht, hat sich das Bild gegenüber den vorherigen Stadien vereinfacht. Außer dem erwähnten Kontinuum, das im folgenden als „W“Spektrum bezeichnet sei, ist nur noch die Benzol-RingEmission bei $2600 \AA$ vorhanden. Ihre Intensität läßt sich durch höhere Stromstärken noch weiter herabsetzen, so daß dann die Intensität des „W“-Spektrums

4 z. B. H. S c hüler u. A. Woeldike, Physik Z. 43, 17 [1942].

5 Vgl. H. S c hü le r u. A. W o eldike, Physik. Z. 42, 390 [1941], Abb. 8, Spektr. 3 a. dominiert. (Es ist weder Helium noch $\mathrm{C}_{2}, \mathrm{CH}, \mathrm{H}_{2}$ sichtbar. Die Spektren „T“, „U“ und „V“ sind vollständig verschwunden.)

Das „W“-Spektrum wird nur bei hohem Dampfdruck des Benzols beobachtet. Die Angabe von $+20^{\circ} \mathrm{C}$ für die Temperatur des Bades entspricht aber nicht mehr dem wahren Dampfdruck am Verdampfungsort, denn auch bei dieser Temperatur des Wasserbades ist das Benzol noch in festem Zustand. Der Kontakt der Substanz mit den Gefäßwänden ist nicht stark genug, um die Temperatur des Bades auf das Benzol zu übertragen ${ }^{6}$. Sicher jedoch ist der Dampfdruck in der positiven Säule größer als im Stadium der Abb. 1, Spektr. c, aber er wird nicht höher als etwa $5 \mathrm{~mm}$ sein, da die Entladung noch keine Fadenbildung zeigt, die bei Drucken größer als $5 \mathrm{~mm}$ beobachtet wird. Für die Beurteilung des „W“-Spektrums sind 3 Beobachtungen wichtig:

a) Es treten keine kleineren Bruchstücke wie $\mathrm{C}_{2}$, $\mathrm{CH}$ und $\mathrm{H}_{2}$ gleichzeitig auf. Es scheint also trotz der größeren Stromstärke in diesem Stadium keine Zerstörung der Benzolmoleküle vorzuliegen.

b) Weiter läßt sich zeigen, daß das „W“-Spektrum nicht identisch ist mit der UV-Emission von Diphenyl; dieses hat wohl auch ein Kontinuum, aber es liegt um etwa $300-400 \AA$ nach Violett verschoben. Eine Diphenylbildung in der Entladung kann also ausgeschlossen werden.

c) Das „W“-Spektrum kann nicht als ein Auslauf der Benzolringemission von $2600 \AA$ angesehen werden, weil sich unter geeigneten Bedingungen zwischen diesen beiden Spektren ein deutliches Minimum abhebt und das Maximum von „W“ unabhängig von der Intensität der Ringemission seine Lage nicht ändert.

Wenn das „W“-Spektrum einer Anregung des unzerstörten Benzolmoleküls zuzuschreiben ist, dann könnte es sich prinzipiell um einen Triplett-SingulettÜbergang handeln. Dieser liegt nach den Befunden der Absorption und der Phosphoreszenz ${ }^{7}$ in dem gleichen Spektralbereich. (Lage des Triplett-Terms $v=29400 \mathrm{~cm}^{-1}, \lambda=3400 \AA$.) $\mathrm{Die}$ vorliegenden Beobachtungen in der Glimmentladung lassen aber bisher keine Struktur in diesem Kontinuum (,W“-Spektrum) erkennen, so daß ein Beweis, ob es sich um den gleichen Übergang handelt wie bei den Banden der

6 Das Benzol schmilzt auch bei höherer Temperatur des Bades noch nicht, weil es sich, wie oben erwähnt, um ein strömendes System handelt, das eine starke Verdampfung des Benzols voraussetzt, wodurch ihm dauernd Wärme entzogen wird.

7 G. N. L e w is u. N. K a s h a, J. Amer. chem. Soc. 67, 994 [1945]; H. Shull, J. chem. Physics 17, 295 [1949]. 
Phosphoreszenz, nicht erbracht werden kann. Schwer verständlich wäre ja das Auftreten eines TriplettSingulett-Überganges in der Glimmentladung, weil der Triplett-Zustand nach den Messungen der Phosphoreszenz eine Lebensdauer von $7 \mathrm{sec}$ hat ${ }^{8}$.

Die Versuchsreihe, deren Ergebnisse in Abb. 1 wiedergegeben sind, zeigt für den Fall des Benzols, daß es möglich ist, die Anregungsbedingungen in der positiven Säule systematisch zu verändern. Angefangen von den Anregungen durch die hohen Elektronengeschwindigkeiten der Helium-Entladung bis zu den wesentlich tiefer liegenden Elektronengeschwindigkeiten der reinen Benzolentladung herab, zeigt das Benzol verschiedene Spektren, von denen ein jedes nur bei einer bestimmten Elektronengeschwindigkeitsverteilung erscheint. Da die Elektronengeschwindig-

8 D. S. M c Clure, J. chem. Physics 17, 905 [1949]. keitsverteilung abhängig ist von dem Dampfdruck des Benzols und der benutzten Stromstärke, braucht man bei vorgegebener Stromstärke nur die Temperatur des Benzols einzustellen, um reproduzierbar die verschiedenen Spektren zu erhalten. So konnten die vier neuen Spektren in der Benzol-Glimmentladung isoliert werden. Es ist verwunderlich, daß die relativ kleinen Änderungen in der Elektronengeschwindigkeitsverteilung solche ausgesprochenen Differenzierungen im Leuchten des Benzols hervorrufen. Da in der Versuchsreihe offensichtlich alle Stufen von der vollständigen Zerstörung des Benzols (2-atomige Bruchstücke) bis zum Leuchten der reinen Benzolentladung durchlaufen werden, müssen beim Benzol verschiedene, voneinander getrennte Zerfallsmechanismen existieren, bei denen die Träger der verschiedenen Spektren erzeugt und angeregt werden.

\section{NOTIZEN}

\section{Versuche zur Polarisation des Sternenlichtes}

Von W. Hanle und H.S cherer

Physikalisches Institut der Justus-Liebig-Hochschule Gießen

(Z. Naturforschg. 6 a, 165-166 [1951]; eingeg. am 4. März 1951)

Nach neueren Beobachtungen ist das Licht von Sternen in bestimmten Himmelsgegenden polarisiert ${ }^{1}$. Es handelt

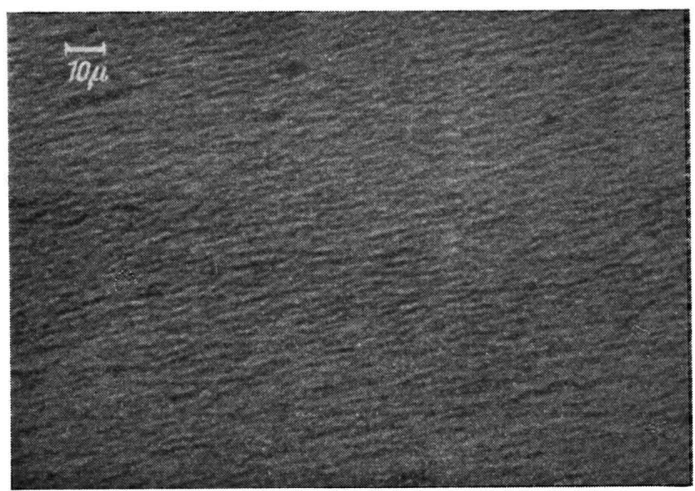

Abb. 1. Mikroaufnahme einer dichroitischen Eisenoxydstaubschicht.

sich dabei nicht um eine primäre Polarisation des von den Sternen emittierten Lichtes. Dieses wird vielmehr beim Durchgang durch den interstellaren Raum polarisiert. Man

1 Lyman Spitzer, jr. u. John W. Tukey, Science [New York] 109, 461 [1 $\left.{ }^{n} 49\right]$; Leverett D avis, jr. u. J e s s e L. Greenstein, Physic. Rev. 75, 1605 [1949]. nimmt an, daß dieser Dichroismus durch längliche Eisenpartikelchen im interstellaren Raum verursacht wird, deren Achsen in eine gemeinsame Richtung zeigen, und welche im Magnetfeld des interstellaren Raumes ausgerichtet werden. Ein Bericht von J o o s 2 veranlaßt uns, eine Beobachtung mitzuteilen, die wir schon vor längerer Zeit gemacht und vorgeführt haben.

Läßt man vor einer Glasplatte in Luft einen Bogen zwischen 2 Eisenelektroden in einem schwachen Magnetfeld brennen, so schlagen sich die Eisenteilchen auf der

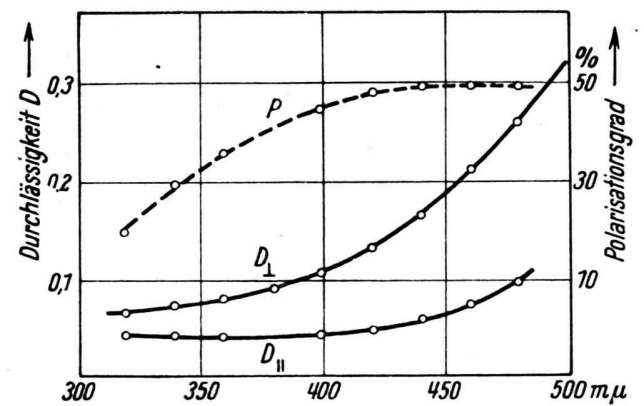

Abb. 2. Durchlässigkeit und Polarisationsgrad einer dichroitischen Eisenoxydstaubschicht.

Glasplatte nieder. Da der Rauch des Bogens aus dem ferromagnetischen kubischen Eisenoxyd $\gamma-\mathrm{Fe}_{2} \mathrm{O}_{3}$ besteht, bilden sich perlschnurartige Ketten, die im Magnetfeld eine einheitliche Orientierung erfahren, wie die Mikroaufnahme Abb. 1 zeigt. Die so im Magnetfeld hergestell-

2 G. J o o s, S.-B. math.-naturwiss. Abt. bayr. Akad. Wiss. vom 7. Juli 1950 . 